\title{
LA EDUCACIÓn Como Superación de la Contraposición entre el Deseo Personal de Bienestar, el Deseo de los Demás y el Derecho, desde Hegel
}

Por: Lyda P. Gil*

Resumen

El presente escrito pretende realizar una aproximación a cómo en medio del devenir propio de las contraposiciones, que surgen en el movimiento dialéctico de Hegel, especialmente en el movimiento del espíritu objetivo, se da una superación que implica una reconciliación de los contrarios. Una superación que en Hegel es expresada de forma pedagógica, es decir, el espíritu en medio de su movimiento dialéctico, realiza un camino pedagógico que le permite superar la contraposición. Contraposición que en un primer momento puede ser entendida como negativa, pero en medio del caminar se convierte en necesaria, pues aunque implica de alguna forma una determinación del espíritu, es el elemento clave para poder desarrollarse y llegar al momento del en sí y para sí de Hegel. Por esta razón, es que se analizará cómo la educación es el movimiento que permite la superación que surge del devenir propio de los intereses personales.

\section{Palabras Claves}

Derecho, educación, superación, devenir, contraposición, espíritu objetivo, moralidad, eticidad.

\begin{abstract}
Within the becoming of antithetic terms in the Hegel's dialectic philosophy, specifically in the deployment of the Objective Spirit, there occurs the overcoming of such antithesis through the reconciliation of the opposite in a pedagogical manner. This outwardly negative antagonism turns into a necessary way-through although it's a determination of the spirit- as to develop into the Absolute in and for itself. Education overcomes the becoming of personal interests.
\end{abstract}

\section{Key words}

Law, Education, Overcoming, Becoming, Antagonism, Objective Spirit, Morality, Ethicity.

\section{Artículo:}

Recibido, 16 de noviembre de 2006; aprobado, 28 de febrero de 2007 


\section{Introducción}

Hegel en su obra Fundamentos de la filosofía del derecho expone el movimiento del espíritu objetivo, en este texto formula una serie de argumentos que buscan mostrar cómo, aunque a los intereses del individuo se les contrapongan los intereses de los demás y del derecho, es posible lograr una superación de esta contraposición. Es decir, Hegel concibe que puede darse una reconciliación de los contrarios, pues ellos devienen de ellos mismos. Para tal fin, Hegel plantea diversos caminos, entre los cuales se puede resaltar el de la educación, el cual es de interés para el análisis del presente escrito. La educación no es un tema que se encuentre trabajado detalladamente como tal por Hegel, pero es el medio que a mi parecer logra encaminar todo el problema de la superación de la contraposición. Pues el espíritu realiza un camino pedagógico de superación o de realización, en el que la negatividad va a jugar un papel esencial. Incluso la educación en un primer momento podría llegar a concebirse como negativa, en la medida en que trata de determinar al espíritu, para lograr su realización, pero este paso por la negatividad es el que permite la formación del espíritu.

Este escrito se dividide en tres partes: en la primera parte, se expondrá de forma breve en qué consiste el movimiento dialéctico de Hegel y cómo éste le sirve para superar las diversas contraposiciones que se presentan en el desarrollo del espíritu objetivo. En la segunda, se esbozaran algunos elementos generales de la eticidad como el momento del en y para sí, en la que se da la educación como la forma de superación de la contraposición entre el mi bienestar y el de los demás. Y por último, una conclusión en la que se plantea cómo la educación es el momento de superación de las contraposiciones.

\section{En qué consiste el movimiento dialéctico de Hegel: a partir de la explicación del movimiento de la voluntad}

La primera tarea que Hegel desarrolla es un análisis del concepto de voluntad, analizando con ésta la universalidad, la particularidad y la individualidad de la voluntad. "La voluntad contiene el elemento de la pura indeterminación o de la pura reflexión del yo en sí, en la que se disuelve toda limitación"' interpretar la voluntad como algo ilimitado y a la vez universal, en palabras de Hegel como el puro pensamiento de sí mismo. Desde esta perspectiva Hegel plantea que no existiría ninguna diferencia entre el pensamiento y la voluntad.

"Cuando un aspecto de la voluntad es aquí determinado es aquello por lo que la voluntad se determina, o es el aspecto tenido para sí por la representación como la libertad, entonces esto es la libertad negativa o libertad del entendimiento" ${ }^{2}$. Con esto busca Hegel afirmar que el hombre actúa por autodeterminación, y que esa libertad de entendimiento la que le da un límite a su actuar. Hegel determina a la libertad del entendimiento también como libertad del vacío y manifiesta que cuando ésta es elevada a la pasión devienen en un fanatismo, que busca la destrucción de todo orden social y es ahí en la destrucción donde adquiere existencia.

Con respecto a la particularidad de la voluntad Hegel afirma:

El yo es igualmente el transito de la indeterminación indiferenciada a la diferenciación, al determinar y el poner una deferminación como contenido y objeto, sea este contenido por la naturaleza o a partir del concepto del espíritu. Por este ponerse a sí mismo como determinado entra el yo en la existencia general; es el momento absoluto de la finitud o particularización del yo. ${ }^{3}$

Para Hegel lo que afirma la particularidad de la voluntad es la acción del yo que no sólo quiere

1. HEGEL, G.W.F. Fundamentos de filosofía del derecho. "Introducción". Traducción: DIAZ, Carlos. K.H ifing Libertarias Prodhut. Madrid, 1993.p. 100

2. Ibídem.

3. Ibidem. p. 104 
algo sino que es algo en particular, algo particular expresado por Hegel como un contenido y un objeto, que son los que ponen la determinación. Pero la determinación también puede ser comprendida como una negatividad, porque es entendida como una carencia, y es a partir de esta negatividad que es el primer momento de la particularidad- que Hegel plantea un segundo momento, donde la voluntad debe ser ilimitada, es decir no debe buscar algo determinado o particular. Ya no es el "yo quiero algo", sino que busca direccionar la voluntad hacia un fin, lo cual implica en Hegel una posibilidad de elección, en la que la voluntad se determina a partir de sus propias elecciones.

Para Hegel la individualidad es la unidad de la particularidad y la universalidad. Es la autodeterminación del yo. "El yo se determina en cuanto es la relación de la negatividad sobre sí mismo; como tal relación sobre sí es asimismo indiferencia frente a esta determinación, la sabe como suya e ideal, como una simple posibilidad a la que no está vinculado, sino en la que él está porque se pone en ella"4. De esta forma Hegel plantea que el yo no está vinculado a una realidad, sino que el yo por medio de su libertad de voluntad, que oscila entre la particularidad y la universalidad y se consuma en la individualidad, decide participar o ponerse en ella. La individualidad es aquello que no es abstracción sino especulación, sentido común.

\section{Movimiento de la eticidad en el espiritu objetivo y el desarrollo del devenir propio de su contraposición}

Todo el movimiento dialéctico del espíritu objetivo de Hegel gira alrededor de los argumentos anteriormente expuestos. Es decir, Hegel plantea que el espíritu objetivo tiene tres momentos en su desarrollo: en un primer momento se encuentra el derecho abstracto el momento del en sí-. De este primer momento deviene su propia negación la moralidad el momento del para sí-, en el que se busca que se interiorice la ley como lo propio para sí. Por último, la eticidad, que es el momento de la reconciliación de los dos primeros y, por tanto, es el momento del en y para sí. Es este último momento el que plantea más claramente cómo la educación es la superación de la contraposición entre mis deseos de bienestar y los deseos de los demás. Y es partir de él que trataré de argumentar las razones por las cuales la educación logra dicho fin.

La idea de eticidad en Hegel parte de la idea de la libertad como la que se encarga de darle unidad a la autoconciencia, que es en Hegel ser ético. De modo que en la eticidad busca afirmar la voluntad conforme al concepto de acuerdo al despliegue dialéctico-. Es decir,

La eticidad es la idea de la libertad en cuanto bien viviente que tiene en la autoconciencia su saber, su querer y su realidad, asi como este actuar tiene en el ser ético su base en si y para sí y su fin motor, el concepto de libertad que se ha convertido en mundo existente $y$ en naturaleza de la autoconciencia. $^{5}$

Al darle el carácter de bien viviente a la libertad Hegel busca afirmar que ésta no es estática sino que se mantiene en un movimiento constante, un movimiento que le da su característica de negatividad, porque es la negación en la que se afirma, es decir, el carácter de viviente significa para Hegel movimiento dialéctico. Es por esto que la idea de eticidad es la realización de dicho movimiento o el en sí y para sí de este proceso en el que se encuentra la autoconciencia. Esa autoconciencia de la libertad es la que logra la unidad entre el derecho abstracto, la moralidad y la eticidad. Para Hegel la eticidad, entonces debe ser entendida, por un lado, como una conciencia, pues entiende que cada momento de la idea voluntad- es una totalidad para sí mismo y, a la vez, tiene a la idea como la base de todo proceso y como contenido del mismo. Y por otro lado, como ser "en tanto en cuanto que es saber esta unidad del concepto de la voluntad y de su existencia que es voluntad particular" ${ }^{\prime \prime}$.

4. Ibidem. p. 107

5. HEGEL, G.W.F. Fundamentos de filosofía del derecho. "La eticidad". Traducción: DIAZ, Carlos. K.H iting Libertorias Prodhuf. Madrid, 1993. p. 530

6. Ibídem. p. 532 
Después que Hegel ha definido la idea de eticidad como ser y como conciencia y como el momento en sí y para sí de la libertad, procede a explicar cómo en la idea de la eticidad lo ético es objetivo, para lo cual Hegel afirma que:

Lo éfico objefivo que aparece en el lugar del bien abstracto es, por medio de la subjetividad, la sustancia concreta en cuanto forma infinita. Ella establece, pues, diferencias en si, de este modo determinadas por el concepto, y por ellas tiene lo ético un contenido fijo que es necesario para sí y que es una experiencia elevada por encima de la opinión subjetiva y del capricho: las leyes e instituciones existentes en síy para si. ${ }^{7}$

Con base en la cita anterior, se observa cuál es el movimiento dialéctico de la eticidad, pues parte de un primer momento en el cual lo ético es un bien abstracto subjetivo- que se encuentra en una forma infinita. Es decir, el momento al que Hegel denomina el en sí, donde lo ético tiene que partir de sus diferencias para poder ser determinado por el concepto y establecer su contenido propio. Al establecer lo ético su contenido propio pasa a su segundo momento, el para sí en el cual se encuentra la subjetividad como mi voluntad, en donde por medio de su negación y es por medio de su devenir propio que llega al último momento de este proceso dialéctico, el en sí y para sí tercer momento- que se expresa o se desarrolla en algo concreto, es decir en lo ético. Ese algo concreto son las leyes y las instituciones, que existen para resguardar la libertad $y$, por tanto, es una subjetividad como ser universal, ya que estas leyes e instituciones constituyen lo ético objetivo. En este punto se encuentra, en un primer momento, la relación existente entre la ética y el derecho abstracto, pues ambos consideran a las leyes como objetivas, pero para cada uno de estos las leyes tienen una función y un movimiento especial, pues mientras que en el derecho las leyes se encuentran en un momento abstracto, en la ética se dan por medio de un proceso dialéctico, es decir, se encuentran no en lo abstracto sino en el en sí y para sí de todo el proceso.
Hegel plantea que un objeto del saber es la sustancia ética, dentro del cual se analiza el papel que juegan las leyes como autoridad y cuál es el papel de lo ético en la autoconciencia, manteniendo con ello una relación constante, con lo planteado anteriormente, cuando se afirmó que la eficidad podría ser entendida como ser y como conciencia. En lo concerniente al papel de las leyes como autoridad, Hegel afirma:

En esta autoconciencia real la sustancia es sabedora de sí misma y por tanto objeto del saber. Para el sujeto la sustancia ética, sus leyes y fuerzas, tienen por una parte la condición de ser, en el sentido más elevado de la independencia, una autoridad $y$ un poder absolutos, infinitamente más fuertes que el ser de la naturaleza.

Hegel realiza en este punto un paralelo entre las leyes y las fuerzas de la naturaleza y la autoridad de las leyes éticas. De las primeras Hegel afirma que se dan su ser a sí mismas y que a la vez, tienen conciencia de sí como una naturaleza particular que se rige por la autoridad de sus leyes, que son contingentes. Mientras que las segundas son más elevadas porque expresan la racionalidad de una manera no externa y aislada, sino como interna e intima y esto le da a la eticidad su carácter de ser.

Por otro lado, Hegel plantea que desde una perspectiva de eticidad las determinaciones sustanciales 9 son como deberes para el individuo, "vinculantes para la voluntad del individuo, el cual se distingue de ellos como lo subjetivo y en sí indeterminado o como lo particularmente determinado, y de este modo se halla en relación con ellas como con lo suyo sustancial"10. La perspectiva desde la cual Hegel concibe en este punto los deberes se centra en la teoría ética, apartándose con ella de la teoría moral. Ya que considera que esta última se centra en la subjetividad, mientras que la primera tiene como principio el ser objetiva, porque se encuentra en el último momento del movimiento dialéctico de la libertad, en el que se da un "desarrollo sistemático del ámbito de la necesidad ética" "1", en la que se

7. Ibidem.

8. Ibidem.

9. Las leyes y la autoconciencia

10. Ibidem. p. 542

11. Ibidem. 
considera que el sujeto se relaciona con los deberes por su diferencia con ellos, ya que permite realizar una vinculación con la voluntad.

Hegel considera que otra diferencia que se da entre la teoría ética y la moral, en lo referente a la teoría de los deberes, es que en el campo de la ética las determinaciones "resultan de relaciones necesarias, por lo cual se concluye aquí y no se añade a cada una de ellas la proposición 'en consecuencia de esta determinación constituye para los seres humanos un deber ${ }^{\prime \prime \prime 12}$. Esta teoría del deber, para Hegel, obtiene la materia del despliegue de las relaciones que establece cada individuo, por esta razón las considera existentes-y por ello se hacen presentes en sí cada una de las representaciones, los principios, pensamientos, instintos y todo aquello que constituye al sujeto en el momento de poner en juego su propia libertad. Pues la libertad se constituye de todo lo que es el ser humano en esencia, por eso las relaciones que establece un individuo son esenciales y reales para el Estado.

Además, Hegel considera que el deber, en cuanto obligación para el sujeto, se puede considerar como una limitación a la luz de la subjetividad, pues, choca con el sujeto que es una subjetividad indeterminada y que es una voluntad moral que se rige por impulsos. Estos son los que determinan su bien indeterminado por elección, mas no por un deber ser. Pero en la eticidad los deberes no son una limitación sino una liberación para el sujeto y en ese sentido los deberes son considerados a la luz de la objetividad.

Para Hegel lo ético, al reflejarse en aquello que tiene un carácter individual determinado por la naturaleza, es decir, el en sí y para sí, se puede considerar como la virtud, que muestra la adecuación que hace el sujeto de los deberes a las relaciones que se le presentan en su vida cotidiana, donde lo ético es idéntico a la personalidad particular, y Hegel considera que la virtud que refleja este relacionarse es la honradez. Para ampliar un poco más esta idea Hegel se pregunta "qué debería hacer, cuáles son los deberes que debería cumplir el hombre para ser virtuoso"13. Para Hegel estas preguntas son fáciles de solucionar si se encuentra en una comunidad ética, en la cual hay que hacer aquello que está pre-trazado, expresado y conocido en cada una de las relaciones que establece el sujeto. Relaciones en las que "la honradez es lo universal que se puede exigir, en parte jurídicamente y en parte éticamente ${ }^{\prime \prime 14}$.

Además, la sustancia ética en Hegel puede ser entendida como espíritu, pues se concibe a la eticidad como algo que emerge en la costumbre, lo cual le permite al individuo identificarse con la realidad, pues su actuar se encuentra inmerso en un actuar universal que le permite reconocer su realidad exterior. Por ello Hegel afirma que "el hábito de los mismos como segunda naturaleza que se pone en lugar de la primera voluntad simplemente natural y que es el alma penetrante, el significado y la realidad de su existencia, el espíritu en cuanto mundo viviente y existente cuya sustancia es así sólo como espíritu" ${ }^{\prime 15}$. Con ello el individuo desarrolla una conciencia que es unicidad, no sólo interior sino también exterior, y a la vez identidad con la realidad, diferente a la autoconciencia de la moralidad, en la cual hay tan sólo una interioridad que se refleja en otros pero que vuelve en $\mathrm{si}^{16}$. Hegel afirma que

La sustancialidad ética ha alcanzado de esta manera su derecho y éste su validez, de suerte que en ella ha desaparecido la obstinación y la propio conciencia moral del individuo que fuera para si y que se le contrapusiera, puesto que el carácter ético sabe que su finalidad motora es lo universal inmóvil, pero abierto en sus determinaciones a la racionalidad efectiva, $y$ reconoce que su dignidad, así como toda conciencia de los fines particulares, se funda en ello y las tiene realmente en ello. ${ }^{77}$

En el derecho abstracto Hegel había planteado que el derecho se encontraba en una forma en sí, pero en la eticidad el derecho es contemplado

\footnotetext{
12. Ibidem.

13. Ibídem.

14. Ibidem. p. 546

15. Ibídem. p. 550

16. Cfr lbidem. p. 551 Comentario

17. lbidem. p. 552
} 
como en sí y para sí, en el cual el carácter ético lo determina como inmóvil y abierto a la racionalidad de sus determinaciones. Con esto Hegel determina qué es lo subjetivo y qué lo real, en donde lo subjetivo es la forma absoluta de las determinaciones y lo real es la diferencia de la forma de la determinación en cuanto objeto, fin y poder.

Por tanto, la eticidad considerada como la verdad de la certeza subjetiva es en la que el sujeto alcanza su determinación. Pues, "el derecho de los individuos a su determinación subjetiva a la libertad tiene su cumplimiento en el hecho de que ellos pertenecen a una realidad ética, pues la certeza de su libertad tiene su verdad en tal objetivamente, y ellos poseen realmente su propia esencia en lo ético, su universalidad interior" ${ }^{\prime 18}$.

Lo anterior implica que la mejor forma de educar a un niño es hacerlo hijo de su tiempo, o como afirmaría un pitagórico "ciudadano de un Estado de leyes buenas". En el Estado ético el derecho de la particularidad, que contiene la sustancialidad ética, representa la unión entre la voluntad universal y la voluntad particular, pues, según Hegel, es en la particularidad en la que se da el modo fenoménico externo en donde emerge lo ético. Desde esta panorámica es, para Hegel, necesario determinar la unidad que existe entre el deber y el derecho. "En esta identidad de la voluntad universal y de la particular se anulan, pues, deber y derecho, y por medio de lo ético tiene el ser humano derechos en la medida en que tiene deberes y deberes en la medida que tiene derechos ${ }^{\prime \prime 19}$, donde es encuentra la existencia de la libertad.

Entonces, la mayor diferencia entre lo ético, el derecho abstracto y la moral, versa en: el derecho abstracto los derechos son para mí mientras que los deberes son para los otros y no existe una concatenación de los dos. Y en la moral están unidos los deberes con el derecho únicamente en la medida en que el derecho hace parte de mí querer. Mientras que para lo ético existen derechos en la medida en que existen deberes y viceversa es decir, los unos dependen de los otros para ser y para que puede existir en el individuo una libertad de decisión.

\subsection{La eticidad en relación con el espíritu de la familia y el espíritu del pueblo}

Un ejemplo de lo anterior se distingue en que Hegel realiza una diferenciación entre lo que es el espíritu de la familia y el espíritu del pueblo o el Estado. Una diferencia es que en la familia lo que existe son "personas libres, autónomas, que velan por si $^{\prime \prime 20}$, mientras que en el estado existe una dependencia, en la que el Estado es el encargado de velar por la libertad de las personas. Pero la relación que existe entre el Estado y la familia es que la sustancia ética es su espíritu real.

Al igual que el espíritu objetivo y la voluntad se desarrollan de acuerdo a una serie de momentos, la eticidad también tiene unos momentos que le permiten su despliegue. El primer momento es la familia, en la que se da un vínculo entre la universalidad comunitaria y la libertad. Es en la familia por medio del matrimonio que se realiza la libertad subjetiva, porque en el matrimonio se da una entrega subjetiva que permite por medio del amor dar una solución al problema de los intereses diversos. El segundo momento es el de la sociedad civil, en la que se contraponen los intereses comunitarios con los de la familia. Lo que implica que "la familia se disuelve natural y esencialmente merced al principio de personalidad en una pluralidad de familias que, en general, se comportan mutuamente como personas autónomas concretas y, por tanto, externas entre si" $^{\prime 21}$. El tercer momento es el del Estado, en el que se reconcilian los dos momentos anteriores y además, es el Estado el encargado de velar por la libertad de las personas. En razón de la relación que existe entre el estado y la familia, es que la sustancia ética es su espíritu real.

De acuerdo con lo anterior, se puede plantear que Hegel ve en el movimiento dialéctico como 
necesario el paso por la negatividad, ya que la libertad no depende de un individuo aislado, sino que la libertad se desarrolla a través del encuentro con otros intereses que se le contraponen, que le permiten al hombre ser consciente de su bienestar como un bien común, es decir, se realizan los intereses en el encuentro con el otro. Por ende, en Hegel no se puede eliminar el conflicto, pues el motor del espíritu absoluto es la negatividad.

\section{Conclusión: La educación como momento de superación de la contraposición}

Con todo lo anterior se nos abre la panorámica para plantear que la superación del conflicto entre mi deseo de bienestar y el deseo de bienestar de los demás es la educación. Cuando se quiere hablar de la educación en Hegel, lo primero que hay que tener en cuenta es Hegel con sus planteamientos realizar una crítica a aquellas teorías que consideran al hombre únicamente en su estado de naturaleza. Pues para Hegel el hombre vive en una relación espiritual, una relación de encuentro e interacción con otros, y es en ella donde realiza su toma de posesión, en la que se sabe a sí mismo como libre, dueño de su vida y se autoreconoce. Por ejemplo, un niño, aunque esté orientado por sus propios instintos, cuando se encuentra inmerso en una cultura se empieza a autocontrolar, es decir, el individuo inicia la construcción de sí a partir de la relación de contraposición que se da entre su autoconciencia y las leyes que regulan el estado. Pues es un autoformase, que le permite al niño hacerse dueño de sí mismo por medio de las relaciones sociales que establece, en un sentido inmediato. Entonces, en Hegel educarse es trabajarse a sí mismo, un autoformarse, un autoconstrucción de sí, un formarse con los otros.

De acuerdo con la anterior, es necesario comprender que, para Hegel, el hombre es un ser natural que vive externo a su concepto el concepto del hombre es la libertad-y sólo por el cultivo de la Bildung $^{22}$ es que logra su realización. Es el concepto de Bildung el que da una imagen de que es la educación en Hegel. En sentido heterónomo ella implica que se toma la materia y se le inculca una forma. Por esto, es que a través de la materia sus propias potencialidades y pasiones- el hombre se hace a sí mismo. Pues es en la materia en la que se construye el espíritu. Es así, como para Hegel una persona es como una semilla que crece y se desarrolla, que tiene una serie de pasiones, que son un momento en la formación del hombre, que se afirma a partir de la negatividad de ésta. A la vez, permiten desarrollar algo interior en el hombre. Por esto la pasión es racional, pues la pasión implica la fuerza vital del hombre. Es por medio de la pasión que Hegel explica el movimiento dialéctico de la formación del hombre.

Por otro lado, en el sistema de necesidades de la sociedad civil la educación es la encargada del reconocimiento real del hombre en tanto en relación con otros. En la sociedad civil las leyes son la que permiten ese reconocimiento, pues es a través de ellas que yo me relaciono con otros y es en esa relación que yo ejerzo mi libertad. Es la educación la que me permite comprender la ley y obedecerla. En la ley se encuentran en juego mis deseos de bienestar y los deseos de bienestar de los demás, y sólo en la medida en que yo comprendo la ley se puede superar la contraposición que se da en el deseo de bienestar, en esa confrontación es que yo me educo y formo mi libertad. Entonces, es la educación

La que le confiere al derecho la existencia para ser reconocido, sabido y querido como universal y a tener validez $y$ realidad objetiva mediadamente $a$ través de este ser sabido y querido. (...) Corresponde a la educación, al pensamiento en cuanto conciencia del individuo en la forma de universalidad, que yo sea tomado como persona universal, en la que todos son idénticos. Así el hombre vale porque es hombre ${ }^{23}$.

En conclusión, aunque a mis intereses se le contrapongan los intereses de los demás y el mismo derecho, es posible lograr una superación de esta contraposición en la medida en que por

22. Bild: que significa imagen. Se toma una figura o forma que define la materia. En el hombre la bildung implica un aprendizaje de la cultura.

23. Ibidem. p. 640 
medio de la educación existe una relación con los otros que me permite reconocerme y reconocer al otro. A la vez, la contraposición con el derecho se supera en la medida en que comprendo la ley y la obedezco, porque hay una interiorización de la misma y la hago parte de mi ser. Esta superación se da plenamente en el Estado, ya que éste es el encargado de velar por la libertad de todos los ciudadanos, que en él se reconocen. 